\title{
Ismar Boas †
}

Ismar Boas starb am 15. März 1938 in Wien. Mit ihm ist einer der großen Koryphäen der medizinischen Wissenschaft dahin gegangen.

Boas wurde am 28. März 1858 geboren und begann seine ärztliche Tätigkeit im Jakre 1882 als praktiscker Arzt in Berlin. Im Jahre 1886 etablierte er sich als Facharzt für Magenkrankheiten. Er gab bald Kurse für dieses Fach, die eine große Bedeutung für die Verbreitung der Kenntnisse der Gastroenterologie hatten. Groß ist die Zahl seiner wissenschaftlichen Veröffentlichungen. Im Jahre 1890 erschien sein Buch über, ,Diagnostik und Therapie der Magenkrankheiten”, das einen großen Erfolg hatte und viele Auflagen erlebte. Dann veröffentlichte er Werke über ,,Darmkrankheiten”, über „,Okkulte Blutungen”, über ,,Ver-stopfungen und deren Behandlung”. Im Jahre 1895 begründete Boas sein ,,Archiv für Verdauungskrankheiten”. Zu den ersten Mitheraus-gebern wählte er dabei die folgenden Herren: v. Noorden, Frieãr. v. Müller, Pawlow, Riegel, Nothnagel, Oítomar Rosenbach, 0. Leichten-

Archiv für Verdauungskrankheiten. Bd. 63. Heft 1/2 (Mai 19E8). 1

-2

Ismar Boas $\dagger$.

stern, Penzoldt, Jul. Schreíber, Max Einhorn, Hemmeter, Stiller, Fleî́ner, Martins, Alb. Mathíeu.

Dieses ,Archiv” hat viel zur weiteren Ent-wicklung unserer Kenntnisse der

Verdauungskrankheiten und ihrer Behandlung beigetragen.

Zahllos sind die wissenschaftlichen Probleme, die Boas bearbeitet und gelöst hat. Im Verein mit C. A. Ewald schuf er das noch jetzt allgemein gebrauchte Probefrühstück und die

Expressionsmethode für die Untersuchung des Magensaftes. Ihm verdanken wir die Kennt-nis von dem häufigen Vorkommen der Milchsäure bei Magenkrebs, ferner der Befund von langen Bazillen (Oppler-Boas), die gewöhnlich das Vorkommen von Milchsäure begleiten; das Fehlen von freier Salz-säure bei Magenkrebs, das schon von v. d. Velden festgestellt worden war, wurde von Boas bestätigt. Er gab die Besorzin-Zuckerprobe zum Nachweis der freien Salzsäure an, sowie die ,,Superaciditas mucosa”, , Colitis ulcerativa':, , ,Magensteifung”, Tropf Mistier für Bektal-ernährung". Weiter entdeckte Boas das Vorkommen von okkulten Blutungen bei peptischen Geschwüren und bei Magen-Darmkarzinomen.

Boas gehört zu denen, die für die Medizin unsterblich sind und seine Leistungen werden die Grundlage für die Arbeit noch vieler kommender Generationen bleiben.

Ich glaube das Leben meines verehrten Freundes nicht besser kennzeichnen zu können, als durch das folgende Zitat aus seiner Ergo-biographie:

„, Praxis und Wissenschaft waren mir Eckpfeiler des Lebens. In jener sah ich immer nur die Kunst des Möglichen. Wunderdiagnosen und Wunderkuren, von denen man so hört oder liest, stehe ich mit größter Skepsis gegenüber, selbst denen, die mir selbst gelegentlich zugeschrieben werden. Mein Hauptziel war und ist, den mir anvertrauten Kranken das Leben und das Sterben zu erleichtern."

,Meine Hauptfreude gait und gilt noch heute meiner wissen-schaftlichen Tätigkeit und der Eörderung des Faches, für dessen Gründung ich mich verantwortlich fühle. Und wenn es in 
unserm dornenvollen Dasein überhaupt ein wahres Glück gibt, so besteht es lediglich darin, hier und da einen bescheidenen Zipfel des geheimnis-vollen Schleiers lüften zu dürfen, der Menschenleben und Menschenleid verhüllt. Um dieses Glückgefühls willen, das zu empfinden mir ver-gönnt war - das Berservat der Forscher und oft ihr einziges - lohnt es sich fürwahr, gelebt und gestrebt, gekämpft und gelitten zu haben."

Die ganze Arztewelt steht trauernd an der Bahre dieses großen Arztes. Max Einhorn, New York. 